\title{
Is day 7 culture necessary for in vitro fertilization of cryopreserved/warmed human oocytes?
}

\author{
Xiangli Niu', Cassie T. Wang ${ }^{2}$, Richard Li $^{2}$, Ghassan Haddad ${ }^{3}$ and Weihua Wang ${ }^{2^{*}}$ (B)
}

\begin{abstract}
Background: Human embryos are usually cultured to blastocyst stage by Day 5 or 6 after insemination. However, some embryos grow slowly and reach blastocyst stage at Day 7. Acceptable live birth rates have been reported after transfer of Day 7 blastocysts resulted from fresh oocyte in vitro fertilization (IVF). It is unknown whether an extended embryo culture to Day 7 is necessary for cryopreserved oocyte IVF to obtain more transferrable blastocysts.

Methods: In this study, 455 oocytes from 57 cycles were warmed, inseminated, and the resulting embryos were cultured by Day 7 to examine blastocyst development after extended culture. Fifty one blastocysts from 16 cycles were biopsied to examine embryo aneuploidies.

Results: It was found that $35.1 \%$ of the cycles had Day 7 blastocysts, and 3.5\% of the cycles had only Day 7 blastocysts. Day 7 blastocysts accounted for $15.6 \%$ of total blastocysts. The proportion of top quality of blastocysts was lower at Day 7 than at Day 5 or 6 . However, no differences were observed on aneuploid blastocyst rates among Days 5, 6 and 7. Similar clinical pregnancy, ongoing pregnancy and embryo implantation rates were obtained after Day 7 blastocyst transfer as compared with Day 5 or 6 blastocyst transfer.

Conclusion: These results indicate that embryos from oocyte warming cycles should be cultured to Day 7 if they do not reach to blastocyst stage by Day 6 so that number of usable blastocysts can be increased.
\end{abstract}

Keywords: Oocytes, Warming, Blastocysts, Day 7 culture, Aneuploidy

\section{Background}

Oocyte cryopreservation has become one of the most important human assisted reproductive technologies, which provides approaches of fertility preservation for women who want to delay childbearing [1], and for young cancer patients before treatment $[2,3]$. It also facilitates the establishment of donor egg bank [4].

The procedures for in vitro fertilization (IVF) and embryo culture did not have any difference between cryopreserved/warmed oocytes and fresh oocytes. However, recently, it has been found by time-lapsing analysis and morphokinetic that cryopreserved/warmed oocytes had

* Correspondence: wangweihua11@yahoo.com

${ }^{2}$ Prelude-Houston Fertility Laboratory, Houston Fertility Institute, 2500

Fondren Rd., Suite 350, Houston, TX 77063, USA

Full list of author information is available at the end of the article about $1 \mathrm{~h}$ of delayed fertilization and embryo development [5]. Delayed embryo development is very often in human IVF even fresh oocytes are used, which is indicated by a delayed blastocyst formation. Day 7 blastocyst accounts for $\sim 5-8 \%$ of total blastocysts [6]. Although most human IVF laboratories culture human embryos to Day 6, an extended Day 7 culture is necessary for some patients, especially if patients have limited number of oocytes [6-8]. It has been reported that Day 7 blastocysts had lower implantation potential as compared with Day 5/6 blastocysts, however, transfer of Day 7 blastocysts can lead to acceptable live birth rates $[9,10]$.

For cryopreserved oocytes from oocyte bank, oocyte number is very limited, usually $6-8$ per cycle, thus the number of embryos developed to blastocyst stage is much fewer than that from most fresh oocyte IVF cycles. Therefore, it may be necessary to extend embryo culture 
to Day 7 so that slow growing embryos can reach to blastocyst stage. In our clinic, we started to Day 7 culture for all oocyte warming cycles from 2019, and our preliminary data showed that implementation of Day 7 culture for oocyte warming IVF cycles increased overall blastocyst number.

\section{Results and discussion}

In this study, 455 cryopreserved donor oocytes were warmed for 57 recipient cycles. Warmed oocytes were inseminated by intracytoplasmic sperm injection and all embryos were extended to Day 7 culture if they did not reach blastocysts by Day 6. As shown in Table 1, the overall survival, fertilization, cleavage and blastocyst rates were 94.1, 77.3, 94.9 and $56.2 \%$, respectively. Days 5, 6 and 7 blastocysts were 34.3, 50.0 and 15.6\%, respectively. The proportion of blastocysts at Day 7 appears to be higher than that with fresh oocyte IVF $(<5 \%)$ reported previously [6]. We found that 20 cycles (35.1\%) had Day 7 blastocysts, which is almost doubled as compared with fresh oocyte IVF (18.5\%) [9]. These results may indicate that embryo development of cryopreserved oocytes is slower in some cases although we still do not know what caused the slow embryo development. We also found that $3.5 \%$ of the oocyte warming cycles had only Day 7 blastocysts, which was the same as that with fresh oocyte IVF [9].

Day 7 blastocysts had poorer quality as compared with Day 5 or Day 6 blastocysts $[9,11,12]$. The similar results were also observed in the present study. We found that only $20.7 \%$ of Day 7 blastocysts were top quality of blastocysts with both good inner cell mass (ICM) and good trophectoderm (TE), which was significantly lower than those at Day $5(73.4 \%)$ or Day 6 (57.0\%). However, we found that $41.4 \%$ of Day 7 blastocysts still have either a good ICM or a good TE. Although it is not very clear

Table 1 Laboratory outcomes after cryopreserved/warmed oocyte IVF and extended embryo culture to Day 7

\begin{tabular}{ll}
\hline No. of warming cycle & 57 \\
No. of oocytes warmed & 455 \\
Mean ages of recipients & $42.8 \pm 6.3$ \\
Mean No. of oocytes warmed & $7.98 \pm 1.87$ \\
No. of oocytes survived & $428(94.1 \%)$ \\
No. of oocytes fertilized & $331(77.3 \%)$ \\
No. of oocytes cleaved & $314(94.9 \%)$ \\
Total No. of blastocysts & $186(56.2 \%)$ \\
At Day 5 & $64(34.4 \%)$ \\
At Day 6 & $93(50.0 \%)$ \\
At Day 7 & $29(15.6 \%)$ \\
No. of cycles with Day 7 blastocysts & $20(35.1 \%)$ \\
No. of cycles with only Day 7 blastocysts & $2(3.5 \%)$ \\
\hline
\end{tabular}

which (ICM or TE) is more important for a blastocyst to become a live birth, a good ICM [13], or good TE [14] is necessary for a blastocyst implantation.

Although previous studies found that more Day 7 blastocysts were aneuploid as compared with Day 5 or Day 6 blastocysts, suggesting that slow growing embryos may be resulted from oocyte aneuploidy $[6,9,11,12]$. In the present study, 16 cycles had blastocyst biopsy for preimplantation genetic testing for aneuploidies (PGT-A), and a total of 51 blastocysts (13 at Day 5, 31 at Day 6 and 7 at Day 7) were biopsied. The euploid blastocyst rates were $53.9,61.3$ and $57.1 \%$, respectively, with no statistical difference being observed among Days 5, 6 and 7 blastocysts. These results may indicate that oocytes quality is different from these studies. In the present study, donor oocytes were used while autologous oocytes at various ages were used in the previous studies $[9,11,12]$. Therefore, slow growing embryos from cryopreserved donor oocytes may not be due to oocyte quality, but due to the procedures of oocyte cryopreservation and warming, which results in delayed embryo development in some cases.

In the present study, there were 35 fresh blastocyst transfers and 16 frozen embryo transfer (FET). There were no statistical differences between fresh blastocyst transfer and FET in terms of clinical pregnancy rates (60.0 vs $56.3 \%$ ), ongoing pregnancy rates (54.3 vs $50.0 \%$ ) and embryo implantation rates (53.9 vs $58.8 \%$ ).

Acceptable live birth rates have been obtained after Day 7 blastocyst transfers [6-10]. However, all of the data in these previous reports were from autologous and fresh donor oocyte IVF. As shown in Table 2, in the present study with frozen donor eggs, we did not find the difference among Days 5, 6 and 7 blastocyst transfer in terms of positive $\beta$-hCG rate, clinical pregnancy rate, ongoing pregnancy rate and implantation rate between days 5, 6 and 7 blastocyst transfer. Three patients had Day 7 blastocyst transfer (one with a euploid blastocyst after PGT-A, and two with no PGT-A tested blastocysts), all three were clinical pregnant and 2 are ongoing pregnant. These results indicate that pregnancy rate is also acceptable after transfer of Day 7 blastocysts resulting from frozen/warmed eggs.

For fresh oocyte donation, recipients usually receive many oocytes and high quality of oocytes usually produces enough blastocysts at Days 5 and 6, thus very few laboratory may culture embryos from donor oocytes to Day 7 . This may be the reason that no data is published on the transfer of Day 7 blastocysts resulted from donor oocytes. However, most IVF clinics now switch fresh oocyte to frozen oocyte IVF when donated oocytes are used [15], and 6 oocytes are warmed for each cycle in most cases. This change may require the implementation of extended embryo culture to Day 7 so that more usable/transferrable blastocysts can be obtained for patients. 
Table 2 Comparison of clinical outcomes of Day 5, 6, and 7 blastocyst transfers

\begin{tabular}{lllllll}
\hline $\begin{array}{l}\text { Transfer } \\
\text { categories }\end{array}$ & $\begin{array}{l}\text { No. of } \\
\text { ET }\end{array}$ & $\begin{array}{l}\text { Positive } \beta \text {-hCG } \\
(\%)\end{array}$ & $\begin{array}{l}\text { Clinical pregnancy } \\
(\%)\end{array}$ & $\begin{array}{l}\text { Ongoing pregnancy } \\
(\%)\end{array}$ & $\begin{array}{l}\text { No. of embryos } \\
\text { transferred }\end{array}$ & $\begin{array}{l}\text { Implanted/transferred embryos } \\
(\%)\end{array}$ \\
\hline Day 5 blast & 39 & $26(66.7 \%)$ & $24(61.5 \%)$ & $22(56.4 \%)$ & $1.10 \pm 0.31$ & $24 / 43(55.8 \%)$ \\
Day 6 blast & 9 & $4(44.4 \%)$ & $3(33.3 \%)$ & $3(33.3 \%)$ & $1.0 \pm 0.0$ & $3 / 9(33.3 \%)$ \\
Day 7 blast & 3 & $3(100 \%)$ & $3(100 \%)$ & $2(66.7 \%)$ & $1.33 \pm 0.58$ & $4 / 4(100 \%)$ \\
$P$ value $^{\text {a }}$ & NA & 0.4 & 0.23 & 0.41 & 0.60 & 0.18 \\
\hline
\end{tabular}

${ }^{a}$ No Statistical differences were observed among Days 5, 6 and 7 blastocysts in terms of $\beta$-hCG, clinical pregnancy, ongoing pregnancy rates, mean number of embryos transferred and embryo implantation rates

\section{Conclusions}

In conclusion, our preliminary data with limited case numbers indicate that Day 7 culture is necessary for cryopreserved/warmed oocyte IVF cycles, especially for those cycles with limited number of oocytes being warmed. The proportions of cycles with Day 7 blastocysts and proportions of blastocysts obtained at Day 7 appear to be higher than those observed with fresh oocyte IVF. In addition, our limited data indicate that aneuploid rate with frozen donor oocytes did not differ between Day 7 blastocysts and Days 5/6 blastocysts although quality of Day 7 blastocysts is not as good as quality of Day 5/6 blastocysts. Transfer of Day 7 blastocysts resulted in successful embryo implantation and clinical pregnancy, and both embryo implantation rate and clinical pregnant rate were not different from those with Day 5 or Day 6 blastocyst transfer. Putting together, these data suggest that the implementation of Day 7 culture for those embryos that have not developed to blastocysts by Day 6 is necessary in oocyte warming cycles.

\section{Abbreviations}

FET: Frozen embryo transfer; ICM: Inner cell mass; IVF: In vitro fertilization; PGT-A: Preimplantation genetic testing for aneuploidies; TE: Trophectoderm

\section{Acknowledgements}

Not applicable.

\section{Authors' contributions}

WW conceived the idea of the study. WW, XLN and RL collected and analyzed the data, XLN, CTW and WHW interpreted the results, and wrote the manuscript. CTW, RL and WW performed oocyte cryopreservation and warming, and $\mathrm{GH}$ managed patients and reviewed the manuscript. All authors read and approved the final manuscript.

\section{Funding}

No Funding was received for this study.

\section{Availability of data and materials}

The primary data for this study is available from corresponding author on reasonable request.

\section{Ethics approval and consent to participate}

All patients are recipients who received donated frozen oocytes from our donor oocyte bank. Patients signed written consents for all laboratory and clinical procedures. The data were retrospectively collected from the medical records so the IRB was waived for this study.

\section{Consent for publication}

Not applicable.
Competing interests

The authors declare that they have no competing interests.

\section{Author details}

${ }^{1}$ Research Center for Reproductive Medicine, Reproductive Hospital of Guangxi Zhuang Autonomous Region, Nanning, Guangxi, China.

${ }^{2}$ Prelude-Houston Fertility Laboratory, Houston Fertility Institute, 2500 Fondren Rd., Suite 350, Houston, TX 77063, USA. ${ }^{3}$ Houston Fertility Institute, 2500 Fondren Rd., Suite 350, Houston, TX 77063, USA.

Received: 27 September 2019 Accepted: 14 January 2020

Published online: 18 January 2020

\section{References}

1. Cobo A, García-Velasco JA, Coello A, Domingo J, Pellicer A, Remohí J. Oocyte vitrification as an efficient option for elective fertility preservation. Fertil Steril. 2016;105:755-64.e8. https://doi.org/10.1016/j.fertnstert.2015.11. 027 Epub 2015 Dec 10.

2. Doyle JO, Richter KS, Lim J, Stillman RJ, Graham JR, Tucker MJ. Successful elective and medically indicated oocyte vitrification and warming for autologous in vitro fertilization, with predicted birth probabilities for fertility preservation according to number of cryopreserved oocytes and age at retrieval. Fertil Steril. 2016;105:459-66.e2. https://doi.org/10.1016/j.fertnstert. 2015.10.026 Epub 2015 Nov 18.

3. Meirow D. Reproduction post-chemotherapy in young cancer patients. Mol Cell Endocrinol. 2000;169:123-31.

4. Graham J, Lim J, Tucker M. Establishing an oocyte cryobank network. Methods Mol Biol. 2017;1568:195-203. https://doi.org/10.1007/978-1-4939-6828-2_14.

5. Cobo A, Coello A, Remohí J, Serrano J, de Los Santos JM, Meseguer M. Effect of oocyte vitrification on embryo quality: time-lapse analysis and morphokinetic evaluation. Fertil Steril. 2017;108(3):491-497.e3. https://doi. org/10.1016/j. fertnstert.2017.06.024.

6. Hammond ER, Cree LM, Morbeck DE. Should extended blastocyst culture include day 7? Hum Reprod. 2018;33:991-7. https:/doi.org/10.1093/humrep/dey091.

7. Hiraoka K, Hiraoka K, Miyazaki M, Fukunaga E, Horiuchi T, Kusuda T, Okano S, Kinutani M, Kinutani K. Perinatal outcomes following transfer of human blastocysts vitrified at day 5, 6 and 7. J Exp Clin Assist Reprod. 2009;6:4.

8. Kovalevsky G, Carney SM, Morrison LS, Boylan CF, Neithardt AB, Feinberg RF. Should embryos developing to blastocysts on day 7 be cryopreserved and transferred: an analysis of pregnancy and implantation rates. Fertil Steril. 2013; 100:1008-12. https://doi.org/10.1016/j.fertnstert.2013.06.021 Epub 2013 Jul 19.

9. Su Y, Li JJ, Wang C, Haddad G, Wang WH. Aneuploidy analysis in day 7 human blastocysts produced by in vitro fertilization. Reprod Biol Endocrinol. 2016;14:20. https://doi.org/10.1186/s12958-016-0157-X.

10. Du T, Wang Y, Fan Y, Zhang S, Yan Z, Yu W, Xi Q, Chen Q, Mol BW, Lyu Q, Kuang $Y$. Fertility and neonatal outcomes of embryos achieving blastulation on day 7: are they of clinical value? Hum Reprod. 2018;33:1038-51. https:// doi.org/10.1093/humrep/dey092.

11. Minasi MG, Colasante A, Riccio T, Ruberti A, Casciani V, Scarselli F, Spinella F, Fiorentino F, Varricchio MT, Greco E. Correlation between aneuploidy, standard morphology evaluation and morphokinetic development in 1730 biopsied blastocysts: a consecutive case series study. Hum Reprod. 2016;31: 2245-54. https://doi.org/10.1093/humrep/dew183 Epub 2016 Sep 2.

12. Kaing A, Kroener LL, Tassin R, Li M, Liu L, Buyalos R, Hubert G, Shamonki M. Earlier day of blastocyst development is predictive of embryonic euploidy across all ages: essential data for physician decision-making and counseling patients. J Assist Reprod Genet. 2018;35:119-25. https://doi.org/10.1007/ s10815-017-1038-8 Epub 2017 Sep 11. 
13. Subira J, Craig J, Turner K, Bevan A, Ohuma E, McVeigh E, Child T, Fatum M. Grade of the inner cell mass, but not trophectoderm, predicts live birth in fresh blastocyst single transfers. Hum Fertil (Camb). 2016;19:254-61 Epub 2016 Sep 14.

14. Hill MJ, Richter KS, Heitmann RJ, Graham JR, Tucker MJ, DeCherney AH, Browne PE, Levens ED. Trophectoderm grade predicts outcomes of singleblastocyst transfers. Fertil Steril. 2013;99:1283-1289.e1. https://doi.org/10. 1016/j.fertnstert.2012.12.003 Epub 2013 Jan 8.

15. Quaas AM, Pennings $G$. The current status of oocyte banks: domestic and international perspectives. Fertil Steril. 2018;110:1203-8. https://doi.org/10. 1016/j.fertnstert. 2018.07.013.

\section{Publisher's Note}

Springer Nature remains neutral with regard to jurisdictional claims in published maps and institutional affiliations.

Ready to submit your research? Choose BMC and benefit from:

- fast, convenient online submission

- thorough peer review by experienced researchers in your field

- rapid publication on acceptance

- support for research data, including large and complex data types

- gold Open Access which fosters wider collaboration and increased citations

- maximum visibility for your research: over $100 \mathrm{M}$ website views per year

At BMC, research is always in progress.

Learn more biomedcentral.com/submissions 